\title{
Mean Sombor index
}

\author{
J. A. Méndez-Bermúdez ${ }^{1, *}$, R. Aguilar-Sánchez ${ }^{2}$, Edil D. Molina ${ }^{3}$, José M. Rodríguez ${ }^{4}$ \\ ${ }^{1}$ Instituto de Física, Benemérita Universidad Autónoma de Puebla, Apartado Postal J-48, Puebla 72570, Mexico \\ ${ }^{2}$ Facultad de Ciencias Químicas, Benemérita Universidad Autónoma de Puebla, Puebla 72570, Mexico \\ ${ }^{3}$ Facultad de Matemáticas, Universidad Autónoma de Guerrero, Carlos E. Adame No.54 Col. Garita, Acapulco Gro. 39650, Mexico \\ ${ }^{4}$ Departamento de Matemáticas, Universidad Carlos III de Madrid, Avenida de la Universidad 30, 28911 Leganés, Madrid, Spain
}

(Received: 4 October 2021. Accepted: 28 October 2021. Published online: 30 October 2021.)

(c) 2021 the authors. This is an open access article under the CC BY (International 4.0) license (www.creativecommons.org/licenses/by/4.0/).

\begin{abstract}
We introduce a degree-based variable topological index inspired on the power (or generalized) mean. We name this new index as the mean Sombor index: $m S O_{\alpha}(G)=\sum_{u v \in E(G)}\left[\left(d_{u}^{\alpha}+d_{v}^{\alpha}\right) / 2\right]^{1 / \alpha}$. Here, $u v$ denotes the edge of the graph $G$ connecting the vertices $u$ and $v, d_{u}$ is the degree of the vertex $u$, and $\alpha \in \mathbb{R} \backslash\{0\}$. We also consider the limit cases $m S O_{\alpha \rightarrow 0}(G)$ and $m S O_{\alpha \rightarrow \pm \infty}(G)$. Indeed, for given values of $\alpha$, the mean Sombor index is related to well-known topological indices such as the inverse sum indeg index, the reciprocal Randić index, the first Zagreb index, the Stolarsky-Puebla index and several Sombor indices. Moreover, through a quantitative structure property relationship (QSPR) analysis we show that $m S O_{\alpha}(G)$ correlates well with several physicochemical properties of octane isomers. Some mathematical properties of the mean Sombor index as well as bounds and new relationships with known topological indices are also discussed.
\end{abstract}

Keywords: degree-based topological index; power mean; Sombor indices; QSPR analysis.

2020 Mathematics Subject Classification: 05C09, 05C92, 26E60.

\section{Preliminaries}

For two positive real numbers $x, y$, the power mean or generalized mean $P M_{\alpha}(x, y)$ with exponent $\alpha \in \mathbb{R} \backslash\{0\}$ is given as

$$
P M_{\alpha}(x, y)=\left(\frac{x^{\alpha}+y^{\alpha}}{2}\right)^{1 / \alpha},
$$

see e.g. [2,17]. $P M_{\alpha}(x, y)$ is also known as Hölder mean. For given values of $\alpha, P M_{\alpha}(x, y)$ reproduces well-known mean values. As examples, in Table 1 we show some expressions for $P M_{\alpha}(x, y)$ for selected values of $\alpha$ with their corresponding names, when available.

Table 1: Expressions for the generalized mean $P M_{\alpha}(x, y)$ for selected values of $\alpha$.

\begin{tabular}{rll}
\hline \hline$\alpha$ & $P M_{\alpha}(x, y)$ & name (when available) \\
\hline$-\infty$ & $P M_{\alpha \rightarrow-\infty}(x, y)=\min (x, y)$ & minimum value \\
-1 & $P M_{-1}(x, y)=\frac{2 x y}{x+y}$ & harmonic mean \\
0 & $P M_{\alpha \rightarrow 0}(x, y)=\sqrt{x y}$ & geometric mean \\
$1 / 2$ & $P M_{1 / 2}(x, y)=\left(\frac{\sqrt{x}+\sqrt{y}}{2}\right)^{2}$ & \\
1 & $P M_{1}(x, y)=\frac{x+y}{2}$ & arithmetic mean \\
& & \\
2 & $P M_{2}(x, y)=\left(\frac{x^{2}+y^{2}}{2}\right)^{1 / 2}$ & root mean square \\
& & \\
3 & $P M_{3}(x, y)=\left(\frac{x^{3}+y^{3}}{2}\right)^{1 / 3}$ & cubic mean \\
$\infty$ & $P M_{\alpha \rightarrow \infty}(x, y)=\max (x, y)$ & maximum value \\
\hline
\end{tabular}


There is a well known inequality for the power mean, namely [3, 9,13]: For any $\alpha_{1}<\alpha_{2}$,

$$
P M_{\alpha_{1}}(x, y) \leq P M_{\alpha_{2}}(x, y),
$$

where the equality is attained for $x=y$.

\section{The mean Sombor index}

A large number of graph invariants of the form

$$
T I(G)=\sum_{u v \in E(G)} F\left(d_{u}, d_{v}\right)
$$

are currently been studied in mathematical chemistry; where $u v$ denotes the edge of the graph $G$ connecting the vertices $u$ and $v, d_{u}$ is the degree of the vertex $u$, and $F(x, y)$ is an appropriate chosen function, see e.g. [4, 15, 16].

Inspired by the power mean and given a simple graph $G=(V(G), E(G))$, here we choose the function $F(x, y)$ in Equation (3) as the power mean $P M_{\alpha}(x, y)$ and define the degree-based variable topological index

$$
m S O_{\alpha}(G)=\sum_{u v \in E(G)}\left(\frac{d_{u}^{\alpha}+d_{v}^{\alpha}}{2}\right)^{1 / \alpha},
$$

where $\alpha \in \mathbb{R} \backslash\{0\}$. We name $m S O_{\alpha}(G)$ as the mean Sombor index.

Table 2: Expressions for the mean Sombor index $m S O_{\alpha}(G)$ for selected values of $\alpha$.

\begin{tabular}{rll}
\hline \hline$\alpha$ & $m S O_{\alpha}(G)$ & index equivalence \\
\hline$-\infty$ & $m S O_{\alpha \rightarrow-\infty}(G)=\sum_{u v \in E(G)} \min \left(d_{u}, d_{v}\right)$ & $S P_{\alpha \rightarrow-\infty}(G)$ \\
-1 & $m S O_{-1}(G)=\sum_{u v \in E(G)} \frac{2 d_{u} d_{v}}{d_{u}+d_{v}}$ & $2 I S I(G)$ \\
0 & $m S O_{\alpha \rightarrow 0}(G)=\sum_{u v \in E(G)} \sqrt{d_{u} d_{v}}$ & $R^{-1}(G)$ \\
$1 / 2$ & $m S O_{1 / 2}(G)=\sum_{u v \in E(G)}\left(\frac{\sqrt{d_{u}}+\sqrt{d_{v}}}{2}\right)^{2}$ & $2^{-2} K A_{1 / 2,2}^{1}(G)$ \\
1 & $m S O_{1}(G)=\sum_{u v \in E(G)} \frac{d_{u}+d_{v}}{2}$ & $2^{-1} M_{1}(G)$ \\
2 & $m S O_{2}(G)=\sum_{u v \in E(G)}\left(\frac{d_{u}^{2}+d_{v}^{2}}{2}\right)^{1 / 2}$ & $2^{-1 / 2} S O(G)$ \\
3 & $m S O_{3}(G)=\sum_{u v \in E(G)}\left(\frac{d_{u}^{3}+d_{v}^{3}}{2}\right)^{1 / 3}$ & $2^{-1 / 3} K A_{3,1 / 3}^{1}(G)$ \\
$\infty$ & $m S O_{\alpha \rightarrow \infty}(G)=\sum_{u v \in E(G)} \max \left(d_{u}, d_{v}\right)$ & $S P_{\alpha \rightarrow \infty}(G)$ \\
\hline
\end{tabular}

Note that for given values of $\alpha$, the mean Sombor index is related to known topological indices: $m S O_{-1}(G)=2 I S I(G)$, where $I S I(G)$ is the inverse sum indeg index [18,19], $m S O_{\alpha \rightarrow 0}(G)=R^{-1}(G)$, where $R^{-1}(G)$ is the reciprocal Randic index [6], and $m S O_{1}(G)=M_{1}(G) / 2$, where $M_{1}(G)$ is the first Zagreb index [7]. Also, it is relevant to stress that the mean Sombor index is related to several Sombor indices: $m S O_{2}(G)=2^{-1 / 2} S O(G)$, where $S O(G)$ is the Sombor in$\operatorname{dex}[5], m S O_{\alpha}(G)=2^{-1 / \alpha} S O_{\alpha}(G)$, where $S O_{\alpha}(G)$ is the $\alpha$-Sombor index [14], and $m S O_{\alpha}(G)=2^{-1 / \alpha} K A_{\alpha, 1 / \alpha}^{1}(G)$, where $K A_{\alpha, \beta}^{1}(G)=\sum_{u v \in E(G)}\left(d_{u}^{\alpha}+d_{v}^{\alpha}\right)^{\beta}$ is the first $(\alpha, \beta)-K A$ index [8]. In addition, the limit cases $m S O_{\alpha \rightarrow \pm \infty}(G)$ correspond with the limit cases $S P_{\alpha \rightarrow \pm \infty}(G)$ of the recently introduced Stolarsky-Puebla index [10].

In Table 2 we report some expressions for $m S O_{\alpha}(G)$ for selected values of $\alpha$ that we identify with known topological indices.

\section{QSPR study of $m S O_{\alpha}(G)$ on octane isomers}

As a first application of mean Sombor indices, here we perform a quantitative structure property relationship (QSPR) study of $m S O_{\alpha}(G)$ to model some physicochemical properties of octane isomers. Here we choose to study the following 
properties: acentric factor (AcentFac), boiling point (BP), heat capacity at constant pressure (HCCP), critical temperature (CT), relative density (DENS), standard enthalpy of formation (DHFORM), standard enthalpy of vaporization (DHVAP), enthalpy of formation (HFORM), heat of vaporization (HV) at $25^{\circ} \mathrm{C}$, enthalpy of vaporization (HVAP), and entropy (S). The experimental values of the physicochemical properties of the octane isomers were kindly provided by Dr. S. Mondal, see Table 2 in Ref. [12].
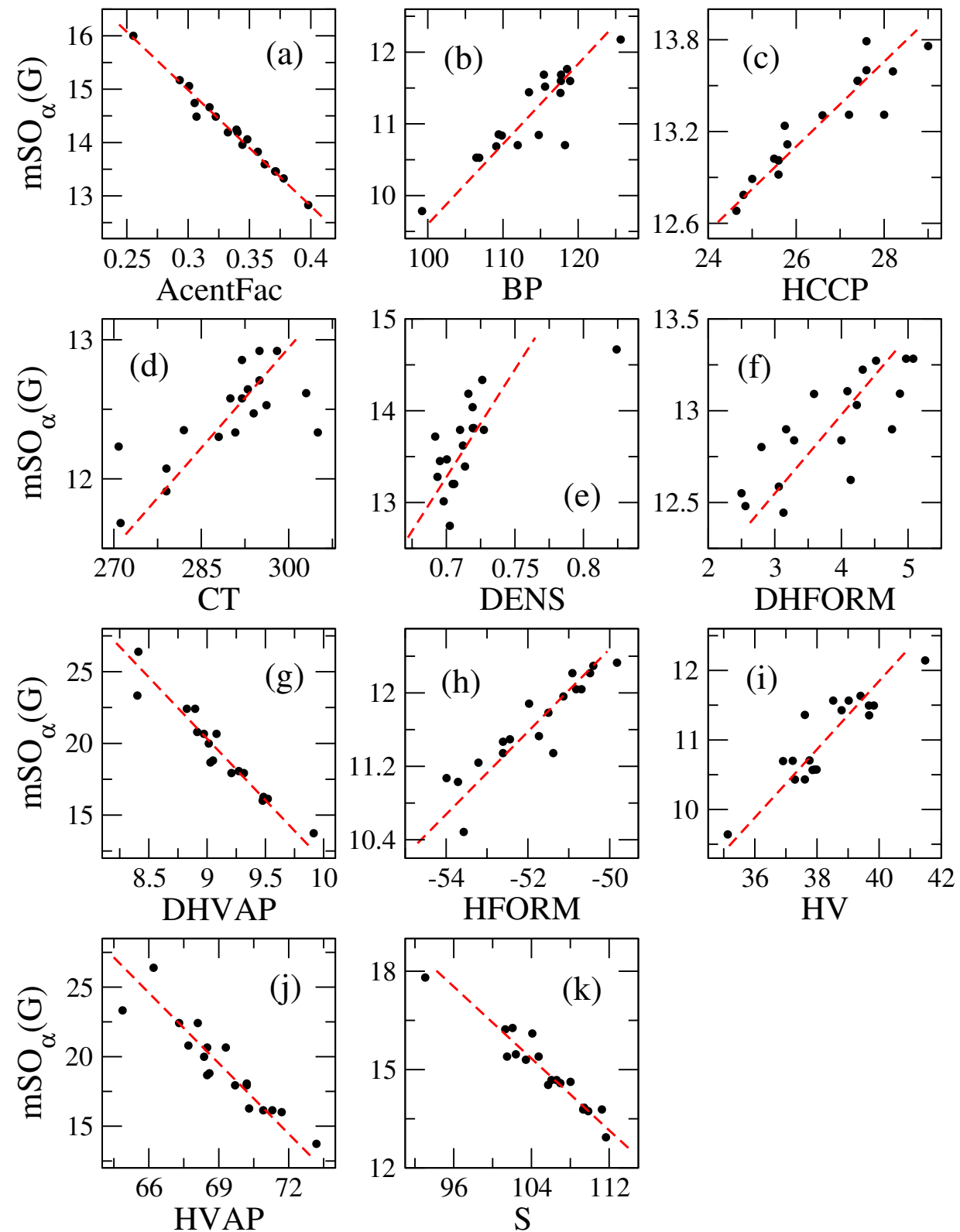

Figure 1: Mean Sombor index $m S O_{\alpha}(G)$ vs. the physicochemical properties of octane isomers, for the values of $\alpha$ that maximize the correlations: (a) $\alpha=0$, (b) $\alpha=-8.19$, (c) $\alpha=-0.87$, (d) $\alpha=-2.05$, (e) $\alpha=-0.53$, (f) $\alpha=-1.28$, (g) $\alpha \rightarrow \infty$, (h) $\alpha=-4.23$, (i) $\alpha \rightarrow-\infty$, (j) $\alpha \rightarrow \infty$, and (k) $\alpha=0.58$. Red dashed lines are the linear QSPR models of Equation (5), with the regression and statistical parameters resumed in Table 3.

In Figure 1 we plot $m S O_{\alpha}(G)$ vs. the physicochemical properties of octane isomers for the values of $\alpha$ that maximize the absolute value of Pearson's correlation coefficient $r$; see Table 3. Moreover, in Figure 1 we tested the following linear regression model

$$
\mathcal{P}=c_{1}\left[m S O_{\alpha}(G)\right]+c_{2},
$$

where $\mathcal{P}$ represents a given physicochemical property. In Table 3 we resume the regression and statistical parameters of the linear QSPR models (see the red dashed lines in Figure 1) given by Equation (5).

From Table 3 we can conclude that $m S O_{\alpha}(G)$ provides good predictions of AcentFac, BP, HCCP, DHVAP, HFORM, HV, HVAP, and $\mathrm{S}$ for which the correlation coefficients (absolute values) are closer or higher than 0.9 . Note that for all these physicochemical properties of octane isomers the statistical significance of the linear model of Equation (5) is far below $5 \%$. Also notice that the mean Sombor index that better correlates (linearly) with the AcentFac is $m S O_{\alpha \rightarrow 0}(G)$, which indeed 
Table 3: For the physicochemical properties $\mathcal{P}$ reported in Figure 1: values of $\alpha$ that maximize the absolute value of Pearson's correlation coefficient $r . c_{2}, c_{1}, S E, F$, and $S F$ are the intercept, slope, standard error, $F$-test, and statistical significance, respectively, of the linear QSPR models of Equation (5).

\begin{tabular}{rrrrrrrr}
\hline \hline property $\mathcal{P}$ & $\alpha$ & $r$ & $c_{2}$ & $c_{1}$ & $S E$ & $F$ & $S F$ \\
\hline AcentFac & 0 & -0.990 & 0.988 & -0.046 & 0.005 & 749.116 & $7.25 \mathrm{E}-15$ \\
BP & -8.19 & 0.886 & 14.115 & 8.946 & 2.929 & 58.126 & $1.00 \mathrm{E}-06$ \\
HCCP & -0.87 & 0.928 & -21.216 & 3.604 & 0.504 & 98.128 & $3.13 \mathrm{E}-08$ \\
CT & -2.05 & 0.717 & 30.048 & 20.859 & 6.788 & 16.934 & $8.10 \mathrm{E}-04$ \\
DENS & -0.53 & 0.702 & 0.134 & 0.042 & 0.022 & 15.518 & $1.17 \mathrm{E}-03$ \\
DHFORM & -1.28 & 0.781 & -26.253 & 2.331 & 0.546 & 24.924 & $1.33 \mathrm{E}-04$ \\
DHVAP & $\infty$ & -0.962 & 11.375 & -0.117 & 0.108 & 196.401 & $2.11 \mathrm{E}-10$ \\
HFORM & -4.23 & 0.912 & -77.705 & 2.220 & 0.530 & 78.903 & $1.39 \mathrm{E}-07$ \\
HV & $-\infty$ & 0.895 & 15.880 & 2.036 & 1.286 & 4.622 & $4.72 \mathrm{E}-02$ \\
HVAP & $\infty$ & -0.921 & 80.550 & -0.592 & 0.813 & 89.724 & $5.81 \mathrm{E}-08$ \\
S & 0.58 & -0.956 & 160.060 & -3.655 & 1.372 & 98.128 & $3.13 \mathrm{E}-08$ \\
\hline
\end{tabular}

coincides with the reciprocal Randić index. Moreover, we found that $|r|$ is maximized when $\alpha \rightarrow \infty$, for DHVAP and HVAP, and when $\alpha \rightarrow-\infty$ for HV. This means that the limiting cases $m S O_{\alpha \rightarrow \pm \infty}(G)$ are also relevant from an application point of view.

\section{Inequalities involving $m S O_{\alpha}(G)$}

Equation (2) can be straightforwardly used to state a monotonicity property for the $m S O_{\alpha}(G)$ index, as well as inequalities for related indices. That is, if $\alpha_{1}<\alpha_{2}$ we have,

$$
m S O_{\alpha_{1}}(G) \leq m S O_{\alpha_{2}}(G),
$$

which implies, for the the first $(a, b)-K A$ index, that

$$
2^{-1 / \alpha_{1}} K A_{\alpha_{1}, 1 / \alpha_{1}}^{1}(G) \leq 2^{-1 / \alpha_{2}} K A_{\alpha_{2}, 1 / \alpha_{2}}^{1}(G), \quad \alpha_{1}<\alpha_{2},
$$

and moreover

$$
2 I S I(G) \leq R^{-1}(G) \leq 2^{-2} K A_{1 / 2,2}^{1}(G) \leq 2^{-1} M_{1}(G) \leq 2^{-1 / 2} S O(G) .
$$

Note that this last inequality involves the inverse sum indeg index, the reciprocal Randic index, the $(a, b)-K A$ index, the first Zagreb index, and the Sombor index. It is fair to acknowledge that the very last inequality in (8) was already included in the Theorem 3.1 of [11].

In what follows we will state bounds for the mean Sombor index as well as new relationships with known topological indices.

We will use the following particular case of Jensen's inequality.

Lemma 4.1. If $g$ is a convex function on $\mathbb{R}$ and $x_{1}, \ldots, x_{m} \in \mathbb{R}$, then

$$
g\left(\frac{x_{1}+\cdots+x_{m}}{m}\right) \leq \frac{1}{m}\left(g\left(x_{1}\right)+\cdots+g\left(x_{m}\right)\right) .
$$

If $g$ is strictly convex, then the equality is attained in the inequality if and only if $x_{1}=\cdots=x_{m}$.

Theorem 4.1. Let $G$ be a graph with medges and $\alpha \in \mathbb{R}$; if $\alpha>1$ then

$$
m S O_{\alpha}(G) \leq \frac{m^{1-1 / \alpha}}{2^{1 / \alpha}}\left(M_{1}^{\alpha+1}(G)\right)^{1 / \alpha},
$$

if $\alpha<1$ and $\alpha \neq 0$ then

$$
m S O_{\alpha}(G) \geq \frac{m^{1-1 / \alpha}}{2^{1 / \alpha}}\left(M_{1}^{\alpha+1}(G)\right)^{1 / \alpha},
$$

and the equality in each bound is attained for a connected graph $G$ if and only if $G$ is regular or biregular. 
Proof. Assume first that $\alpha>1$ then, for $x \geq 0, x^{1 / \alpha}$ is a concave function and by Lemma 4.1 we have

$$
\begin{aligned}
\frac{1}{m} \sum_{u v \in E(G)}\left(\frac{d_{u}^{\alpha}+d_{v}^{\alpha}}{2}\right)^{1 / \alpha} & \leq\left(\frac{1}{2 m} \sum_{u v \in E(G)}\left(d_{u}^{\alpha}+d_{v}^{\alpha}\right)\right)^{1 / \alpha} \\
& =\frac{1}{2^{1 / \alpha} m^{1 / \alpha}}\left(\sum_{u \in V(G)} d_{u}^{\alpha+1}\right)^{1 / \alpha}=\frac{1}{2^{1 / \alpha} m^{1 / \alpha}}\left(M_{1}^{\alpha+1}(G)\right)^{1 / \alpha}
\end{aligned}
$$

Assume now that $\alpha<1$ and $\alpha \neq 0$, then $x^{1 / \alpha}$ is a convex function and by Jensen's inequality we obtain

$$
\begin{aligned}
\frac{1}{m} \sum_{u v \in E(G)}\left(\frac{d_{u}^{\alpha}+d_{v}^{\alpha}}{2}\right)^{1 / \alpha} & \geq\left(\frac{1}{2 m} \sum_{u v \in E(G)}\left(d_{u}^{\alpha}+d_{v}^{\alpha}\right)\right)^{1 / \alpha} \\
& =\frac{1}{2^{1 / \alpha} m^{1 / \alpha}}\left(\sum_{u \in V(G)} d_{u}^{\alpha+1}\right)^{1 / \alpha}=\frac{1}{2^{1 / \alpha} m^{1 / \alpha}}\left(M_{1}^{\alpha+1}(G)\right)^{1 / \alpha}
\end{aligned}
$$

If $G$ is regular or biregular, with maximum and minimum degrees $\Delta$ and $\delta$, respectively,

$$
m S O_{\alpha}(G)=m\left(\frac{\Delta^{\alpha}+\delta^{\alpha}}{2}\right)^{1 / \alpha}=\frac{m^{1-1 / \alpha}}{2^{1 / \alpha}}\left(m\left(\Delta^{\alpha}+\delta^{\alpha}\right)\right)^{1 / \alpha}=\frac{m^{1-1 / \alpha}}{2^{1 / \alpha}}\left(M_{1}^{\alpha+1}(G)\right)^{1 / \alpha} .
$$

If any of these equalities hold, for every $u v, u^{\prime} v^{\prime} \in E(G)$, by Lemma 4.1, we have $d_{u}^{\alpha}+d_{v}^{\alpha}=d_{u^{\prime}}^{\alpha}+d_{v^{\prime}}^{\alpha}$. In particular if we take $u=u^{\prime}$ we have $d_{v}=d_{v^{\prime}}$, so all the neighbors of a vertex $u \in V(G)$ have the same degree. Thus, since $G$ is a connected graph, $G$ is regular or biregular.

In order to prove the next result we need an additional technical result. In [1, Theorem 3] appears a converse of Hölder inequality, which in the discrete case can be stated as follows [1, Corollary 2].

Lemma 4.2. If $1<p, q<\infty$ with $1 / p+1 / q=1, x_{j}, y_{j} \geq 0$ and $a y_{j}^{q} \leq x_{j}^{p} \leq b y_{j}^{q}$ for $1 \leq j \leq k$ and some positive constants a, $b$, then:

$$
\left(\sum_{j=1}^{k} x_{j}^{p}\right)^{1 / p}\left(\sum_{j=1}^{k} y_{j}^{q}\right)^{1 / q} \leq K_{p}(a, b) \sum_{j=1}^{k} x_{j} y_{j}
$$

where

$$
K_{p}(a, b)= \begin{cases}\frac{1}{p}\left(\frac{a}{b}\right)^{1 /(2 q)}+\frac{1}{q}\left(\frac{b}{a}\right)^{1 /(2 p)}, & \text { if } 1<p<2, \\ \frac{1}{p}\left(\frac{b}{a}\right)^{1 /(2 q)}+\frac{1}{q}\left(\frac{a}{b}\right)^{1 /(2 p)}, & \text { if } p \geq 2 .\end{cases}
$$

If $x_{j}>0$ for some $1 \leq j \leq k$, then the equality in the bound is attained if and only if $a=b$ and $x_{j}^{p}=a y_{j}^{q}$ for every $1 \leq j \leq k$.

Theorem 4.2. Let $G$ be a graph with medges, maximum degree $\Delta$ and minimum degree $\delta$, let $0<\alpha<1$, then

where

$$
m S O_{\alpha}(G) \leq \frac{m^{1-1 / \alpha}}{2^{1 / \alpha}} K_{\alpha}\left(M_{1}^{\alpha+1}(G)\right)^{1 / \alpha}
$$

$$
K_{\alpha}^{\alpha}=\left\{\begin{array}{cc}
\alpha\left(\frac{\Delta}{\delta}\right)^{\frac{\alpha-\alpha^{2}}{2}}+(1-\alpha)\left(\frac{\Delta}{\delta}\right)^{\frac{-\alpha^{2}}{2}}, & \text { if } 0<\alpha \leq \frac{1}{2} \\
\alpha\left(\frac{\Delta}{\delta}\right)^{\frac{\alpha^{2}-\alpha}{2}}+(1-\alpha)\left(\frac{\Delta}{\delta}\right)^{\frac{\alpha^{2}}{2}}, & \text { if } \frac{1}{2}<\alpha<1
\end{array}\right.
$$

the equality holds if and only if $G$ is a regular graph.

Proof. For each $u v \in E(G)$ we have

$$
\delta^{\alpha} \leq \frac{d_{u}^{\alpha}+d_{v}^{\alpha}}{2} \leq \Delta^{\alpha}
$$

If we take $x_{j}=d_{u}^{\alpha}, y_{j}=d_{v}^{\alpha}$ and $p=1 / \alpha$ by Lemma 4.2 we have

$$
m^{1-\alpha}\left(m S O_{\alpha}(G)\right)^{\alpha}=\left(\sum_{u v \in E(G)}\left(\frac{d_{u}^{\alpha}+d_{v}^{\alpha}}{2}\right)^{1 / \alpha}\right)^{\alpha}\left(\sum_{u v \in E(G)} 1^{\frac{1}{1-\alpha}}\right)^{1-\alpha} \leq K_{\alpha}^{\alpha} \sum_{u v \in E(G)} \frac{d_{u}^{\alpha}+d_{v}^{\alpha}}{2}=\frac{1}{2} K_{\alpha}^{\alpha} M_{1}^{\alpha+1}(G),
$$


where

$$
K_{\alpha}^{\alpha}=\left\{\begin{array}{cc}
\alpha\left(\frac{\Delta}{\delta}\right)^{\frac{\alpha-\alpha^{2}}{2}}+(1-\alpha)\left(\frac{\Delta}{\delta}\right)^{\frac{-\alpha^{2}}{2}}, & \text { if } 0<\alpha \leq \frac{1}{2}, \\
\alpha\left(\frac{\Delta}{\delta}\right)^{\frac{\alpha^{2}-\alpha}{2}}+(1-\alpha)\left(\frac{\Delta}{\delta}\right)^{\frac{\alpha^{2}}{2}}, & \text { if } \frac{1}{2}<\alpha<1,
\end{array}\right.
$$

and the equality holds if and only if $\delta=\Delta$, i.e., $G$ is regular.

The following inequalities are known for $x, y>0$ :

$$
\begin{aligned}
x^{a}+y^{a}<(x+y)^{a} \leq 2^{a-1}\left(x^{a}+y^{a}\right) & \text { if } a>1, \\
2^{a-1}\left(x^{a}+y^{a}\right) \leq(x+y)^{a}<x^{a}+y^{a} & \text { if } 0<a<1, \\
(x+y)^{a} \leq 2^{a-1}\left(x^{a}+y^{a}\right) & \text { if } a<0,
\end{aligned}
$$

and the equality in the second, third or fifth bound is attained for each $a$ if and only if $x=y$.

Proposition 4.1. Let $G$ be a graph and $\alpha \in \mathbb{R} \backslash\{0\}$, then

$$
\begin{aligned}
2^{-1 / \alpha} S O(G)<m S O_{\alpha}(G) \leq 2^{-1 / 2} S O(G) & & \text { if } 0<\alpha<2, \\
2^{-1 / 2} S O(G) \leq m S O_{\alpha}(G)<2^{-1 / \alpha} S O(G) & & \text { if } \alpha>2, \\
m S O_{\alpha}(G) \leq 2^{-1 / 2} S O(G) & & \text { if } \alpha<0,
\end{aligned}
$$

and the equality in the second, third or fifth bound is attained for each $\alpha$ if and only if each connected component of $G$ is a regular graph.

Proof. If we divide each one of the inequalities in (9) by $2^{a}$ we obtain

$$
\begin{aligned}
2^{-a}\left(x^{a}+y^{a}\right)<\left(\frac{x+y}{2}\right)^{a} \leq \frac{x^{a}+y^{a}}{2} & \text { if } a>1, \\
\frac{x^{a}+y^{a}}{2} \leq\left(\frac{x+y}{2}\right)^{a}<2^{-a}\left(x^{a}+y^{a}\right) & \text { if } 0<a<1, \\
\left(\frac{x+y}{2}\right)^{a} \leq \frac{x^{a}+y^{a}}{2} & \text { if } a<0 .
\end{aligned}
$$

If we take $x=d_{u}^{\alpha}, y=d_{v}^{\alpha}$ and $a=2 / \alpha$; then the previous inequalities give

$$
\begin{array}{cl}
2^{-2 / \alpha}\left(d_{u}^{2}+d_{v}^{2}\right)<\left(\frac{d_{u}^{\alpha}+d_{v}^{\alpha}}{2}\right)^{2 / \alpha} \leq \frac{d_{u}^{2}+d_{v}^{2}}{2} & \text { if } 0<\alpha<2, \\
\frac{d_{u}^{2}+d_{v}^{2}}{2} \leq\left(\frac{d_{u}^{\alpha}+d_{v}^{\alpha}}{2}\right)^{2 / \alpha}<2^{-2 / \alpha}\left(d_{u}^{2}+d_{v}^{2}\right) & \text { if } \alpha>2, \\
\left(\frac{d_{u}^{\alpha}+d_{v}^{\alpha}}{2}\right)^{2 / \alpha} \leq \frac{d_{u}^{2}+d_{v}^{2}}{2} & \text { if } \alpha<0,
\end{array}
$$

and the equality in the second, third or fifth bounds are attained for each $a$ if and only if $d_{u}=d_{v}$. From this we obtain

$$
\begin{aligned}
& 2^{-1 / \alpha}\left(d_{u}^{2}+d_{v}^{2}\right)^{1 / 2}<\left(\frac{d_{u}^{\alpha}+d_{v}^{\alpha}}{2}\right)^{1 / \alpha} \leq 2^{-1 / 2}\left(d_{u}^{2}+d_{v}^{2}\right)^{1 / 2} \quad \text { if } 0<\alpha<2, \\
& 2^{-1 / 2}\left(d_{u}^{2}+d_{v}^{2}\right)^{1 / 2} \leq\left(\frac{d_{u}^{\alpha}+d_{v}^{\alpha}}{2}\right)^{1 / \alpha}<2^{-1 / \alpha}\left(d_{u}^{2}+d_{v}^{2}\right)^{1 / 2} \quad \text { if } \alpha>2, \\
& \left(\frac{d_{u}^{\alpha}+d_{v}^{\alpha}}{2}\right)^{1 / \alpha} \leq 2^{-1 / 2}\left(d_{u}^{2}+d_{v}^{2}\right)^{1 / 2} \quad \text { if } \alpha<0,
\end{aligned}
$$

and the equality in the second, third or fifth bounds are attained for each $a$ if and only if $d_{u}=d_{v}$. The desired result is obtained by adding up for each $u v \in E(G)$.

The following result appears in [13].

Lemma 4.3. If $a_{i}>0$ for $1 \leq i \leq k$ and $r \in \mathbb{R}$, then

$$
\begin{aligned}
& \sum_{i=1}^{k} a_{i}^{r} \geq k^{1-r}\left(\sum_{i=1}^{k} a_{i}\right)^{r}, \quad \text { if } r \leq 0 \text { or } r \geq 1, \\
& \sum_{i=1}^{k} a_{i}^{r} \leq k^{1-r}\left(\sum_{i=1}^{k} a_{i}\right)^{r}, \quad \text { if } 0 \leq r \leq 1 .
\end{aligned}
$$


Proposition 4.2. If $G$ is a graph with $m$ edges, then

$$
\begin{array}{ll}
K A_{\alpha, \beta}(G) \geq m^{1-\beta}\left(M_{1}^{\alpha+1}(G)\right)^{\beta} & \text { if } \beta \leq 0 \text { or } \beta \geq 1, \\
K A_{\alpha, \beta}(G) \leq m^{1-\beta}\left(M_{1}^{\alpha+1}(G)\right)^{\beta} & \text { if } 0 \leq \beta \leq 1 .
\end{array}
$$

Proof. If we take $a_{i}=d_{u}^{\alpha}+d_{v}^{a}$ and $r=\beta$, by Lemma 4.3 we have

$$
\begin{aligned}
& \sum_{u v \in E(G)}\left(d_{u}^{\alpha}+d_{v}^{a}\right)^{\beta} \geq m^{1-\beta}\left(\sum_{u v \in E(G)}\left(d_{u}^{\alpha}+d_{v}^{a}\right)\right)^{\beta}, \quad \text { if } \beta \leq 0 \text { or } \beta \geq 1, \\
& \sum_{u v \in E(G)}\left(d_{u}^{\alpha}+d_{v}^{a}\right)^{\beta} \leq m^{1-\beta}\left(\sum_{u v \in E(G)}\left(d_{u}^{\alpha}+d_{v}^{a}\right)\right)^{\beta}, \quad \text { if } 0 \leq \beta \leq 1 .
\end{aligned}
$$

Given a graph $G$, let us define the mean Sombor matrix $m \mathcal{S M}_{\alpha}(G)$ with entries

$$
a_{u v}:= \begin{cases}\left(\frac{d_{u}^{\alpha}+d_{v}^{\alpha}}{2}\right)^{1 / \alpha}, & \text { if } u v \in E(G), \\ 0, & \text { otherwise } .\end{cases}
$$

One can easily check the following result about the trace of the matrix $m \mathcal{S} \mathcal{M}_{\alpha}(G)^{2}$ :

$$
\operatorname{tr}\left(m \mathcal{S M}_{\alpha}(G)^{2}\right)=\sum_{u v \in E(G)}\left(\frac{d_{u}^{\alpha}+d_{v}^{\alpha}}{2}\right)^{2 / \alpha} .
$$

Denote by $\sigma^{2}$ the variance of the sequence of the terms $\left\{\left(\frac{d_{u}^{\alpha}+d_{v}^{\alpha}}{2}\right)^{1 / \alpha}\right\}$ appearing in the definition of $m S O_{\alpha}(G)$.

Proposition 4.3. Let $G$ be a graph, then

$$
m S O_{a}(G)=\sqrt{\frac{m}{2} \operatorname{tr}\left(m S \mathcal{M}_{\alpha}(G)^{2}\right)-m^{2} \sigma^{2}} .
$$

Proof. By the definition of $\sigma^{2}$, we have

$$
\sigma^{2}=\frac{1}{m} \sum_{u v \in E(G)}\left(\left(\frac{d_{u}^{\alpha}+d_{v}^{\alpha}}{2}\right)^{1 / \alpha}\right)^{2}-\left(\frac{1}{m} \sum_{u v \in E(G)}\left(\frac{d_{u}^{\alpha}+d_{v}^{\alpha}}{2}\right)^{1 / \alpha}\right)^{2}
$$

then using the expression (11) we have

$$
\sigma^{2}=\frac{1}{2 m} \operatorname{tr}\left(m S \mathcal{M}_{\alpha}(G)^{2}\right)-\frac{1}{m^{2}} m S O_{\alpha}(G)^{2},
$$

and the result follows from this equality.

Theorem 4.3. Let $G$ be any graph, then $m S O_{2}(G) \leq M_{1}(G)-M_{2}^{1 / 2}(G)$, where $M_{2}^{1 / 2}$ is the variable second Zagreb index $M_{2}^{\alpha}$ at $\alpha=1 / 2$, and the equality is attained if and only if each connected component of $G$ is a regular graph.

Proof. Let be $\delta, \Delta$ the minimum and maximum degree of $G$, respectively. Let's analyze the behavior of the function

for $\delta \leq x \leq y \leq \Delta$. We have

$$
f(x, y)=(x+y-\sqrt{x y})^{2}-\frac{x^{2}+y^{2}}{2},
$$

$$
\begin{aligned}
\frac{\partial f}{\partial x}(x, y) & =2(x+y-\sqrt{x y})\left(1-\frac{1}{2} \sqrt{\frac{y}{x}}\right)-x=x-3 \sqrt{x y}+3 y-\frac{y \sqrt{y}}{\sqrt{x}} \\
& =\frac{x \sqrt{x}-3 x \sqrt{y}+3 y \sqrt{x}-y \sqrt{y}}{\sqrt{x}}=\frac{(\sqrt{x}-\sqrt{y})^{3}}{\sqrt{x}} \leq 0,
\end{aligned}
$$

so $f$ is a decreasing function for each $y$. Thus, we have $f(x, y) \geq f(y, y)=0$, so

$$
x+y-\sqrt{x y} \geq \sqrt{\frac{x^{2}+y^{2}}{2}},
$$

and the equality is attained if and only if $x=y$. Therefore for any $u v \in E(G)$,

$$
d_{u}+d_{v}-\sqrt{d_{u} d_{v}} \geq \sqrt{\frac{d_{u}^{2}+d_{v}^{2}}{2}}
$$

and the equality is attained if and only if $d_{u}=d_{v}$. The desired result is obtained by adding up for each $u v \in E(G)$. 


\section{Discussion and conclusions}

We have introduced a degree-based variable topological index inspired on the power mean (also known as generalized mean and Hölder mean). We named this new index as the mean Sombor index $m S O_{\alpha}(G)$, see Equation (4). For given values of $\alpha$, the mean Sombor index is related to well-known topological indices, in particular with several Sombor indices.

In addition, through a QSPR study, we showed that mean Sombor indices are suitable to model acentric factor, boiling point, heat capacity at constant pressure, standard enthalpy of vaporization, enthalpy of formation, heat of vaporization at $25^{\circ} \mathrm{C}$, enthalpy of vaporization, and entropy of octane isomers; see Section 3.

We have also discussed some mathematical properties of mean Sombor indices as well as stated bounds and new relationships with known topological indices; see Section 4, where the mean Sombor matrix was also introduced in Equation (10).

Finally, we would like to remark that, in addition to all the known indices that the mean Sombor index reproduces, we discover the indices

$$
m S O_{-\infty}(G) \equiv m S O_{\alpha \rightarrow-\infty}(G)=\sum_{u v \in E(G)} \min \left(d_{u}, d_{v}\right)
$$

and

$$
m S O_{\infty}(G) \equiv m S O_{\alpha \rightarrow \infty}(G)=\sum_{u v \in E(G)} \max \left(d_{u}, d_{v}\right) ;
$$

which, from the QSPR study of Section 3, were shown to be good predictors of the standard enthalpy of vaporization, the enthalpy of vaporization, and the heat of vaporization at $25^{\circ} \mathrm{C}$ of octane isomers. It is fair to mention that several known topological indices include the min/max functions; among them we can mention the min-max (and max-min) rodeg index, the min-max (and max-min) sdi index, and the min-max (and max-min) deg index, introduced in Ref. [19]. However, to the best of our knowledge, the indices $m S O_{ \pm \infty}(G)$ have not been theoretically studied before (for an exception, where the equivalent Stolarsky-Puebla indices have been computationally applied to random networks, see [10]). Thus, we believe that a theoretical study of these two new indices is highly pertinent.

\section{Acknowledgments}

J.A.M.-B. acknowledges financial support from CONACyT (Grant No. A1-S-22706) and BUAP (Grant No. 100405811VIEP2021). E.D.M. and J.M.R. were supported by a grant from Agencia Estatal de Investigación (PID2019-106433GBI00/ AEI/10.13039/501100011033), Spain. J.M.R. was supported by the Madrid Government (Comunidad de Madrid-Spain) under the Multiannual Agreement with UC3M in the line of Excellence of University Professors (EPUC3M23), and in the context of the V PRICIT (Regional Programme of Research and Technological Innovation).

\section{References}

[1] P. Bosch, E. D. Molina, J. M. Rodríguez, J. M. Sigarreta, Inequalities on the generalized ABC index, Mathematics 9 (2021) \#1151.

[2] P. S. Bullen, Handbook of Means and Their Inequalities, Kluwer, Dordrecht, 2003.

[3] B. C. Carlson, Some inequalities for hypergeometric functions, Proc. Amer. Math. Soc. 17 (1966) 32-39.

[4] I. Gutman, Degree-based topological indices, Croat. Chem. Acta 86 (2013) 351-361.

[5] I. Gutman, Geometric approach to degree-based topological indices: Sombor indices, MATCH Commun. Math. Comput. Chem. 86 (2021) 11-16.

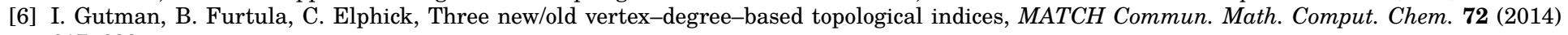
$617-632$

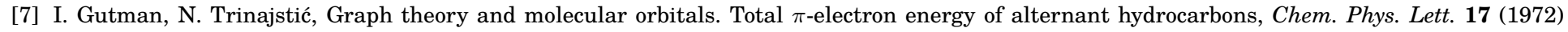
$535-538$.

[8] V. R. Kulli, The $(a, b)-K A$ indices of polycyclic aromatic hydrocarbons and benzenoid systems, Int. J. Math. Trends Tech. 65 (2019) 115-120.

[9] T.-P. Lin, The power mean and the logarithmic mean, Amer. Math. Monthly 81 (1974) 879-883.

[10] J. A. Mendez-Bermudez, R. Aguilar-Sanchez, R. Abreu-Blaya, J. M. Sigarreta, Stolarsky-Puebla index, Discrete Math. Lett. 9 (2022) 10-17.

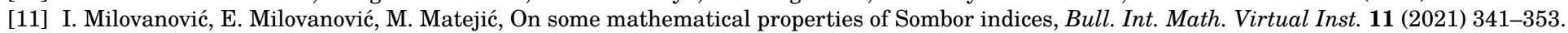

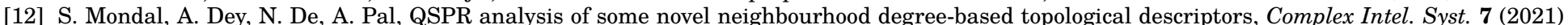
977-996.

[13] B. Ostle, H. L. Terwilliger, A comparison of two means, Proc. Montana Acad. Sci. 17 (1957) 69-70.

[14] T. Réti, T. Došlić, A Ali, On the Sombor index of graphs, Contrib. Math. 3 (2021) 11-18.

[15] J. M. Sigarreta, Bounds for the geometric-arithmetic index of a graph, Miskolc Math. Notes 16 (2015) 1199-1212.

[16] J. M. Sigarreta, Mathematical properties of variable topological indices, Symmetry 13 (2021) \#43.

[17] S. Sykora, Mathematical Means and Averages: Basic Properties, Volume 3, Stan's Library, Castano Primo, 2009.

[18] D. Vukičević, Bond additive modeling 2. Mathematical properties of max-min rodeg index, Croat. Chem. Acta 83 (2010) $261-273$.

[19] D. Vukičević, M. Gašperov, Bond additive modeling 1. Adriatic indices, Croat. Chem. Acta 83 (2010) $243-260$. 